\title{
Modifications of structure and properties of liquids at high temperatures
}

\author{
Alexander Vorontsova and Dmitry Kuts \\ South Ural State University, Pr. Lenina, 76, Chelyabinsk 454080, Russia
}

\begin{abstract}
We present results of the analysis of several structural models of metallic and nonmetallic liquids. Models cover the region of expanded liquid phase and supercritical fluid phase. The goal is to show that there are at least two regions on phase diagram, where liquid phase has essentially different atomic structure. Dense liquid and loose liquid have different atomic and electronic properties, as it could be seen from experiments.
\end{abstract}

\section{Introduction}

It is known, that simple liquids expansion with heating is not an uniform process. Coordination number changes significantly, but the position of the first coordination sphere changes slightly. The biggest effect was observed [1] in liquid alkali metals, where coordination number falls from $8 \ldots 10$ to $3 \ldots 4$ during expansion up to the critical point. Non-uniform expansion leads to the formation of heterogeneous (non-uniform) structure of liquids at low densities. The low-density liquid and high-density liquid should have essentially different properties because of change in interatomic interaction. At high density the repulsive part of the interatomic potential plays the main role, but at low density so does the attractive part. Moreover, in some materials the nature of chemical bonding could change from metallic-like to covalent-like. It was the reason for wellknown problem of Metal-NonMetal phase transition [2]: when does dense metallic liquid transform to non-metalic substance at low densities? The problem is: where the transition takes place - in liquid or in gas phase?

There are a lot of evidences of nonmonotonic changes in properties of "simple" liquids, such as pure liquid metals. Peculiarities were found on the liquid-vapor coexistence curve of $\mathrm{Hg}$ [3], as well as electrical conductivity of $\mathrm{Hg}$ and Cs [3,4], static susceptibility of Cs [5], Knight shift of $\mathrm{Cs}$ and $\mathrm{Hg}[6,7]$, sound propagation in $\mathrm{Hg}[8,9]$, adiabatic thermal coefficient of pressure of Cs [10] and so on. Despite of the extensive evidence of changes in properties and qualitative understanding of expanding process, there is no quantitive criterion of transition in structure and experimental provements of it. Only precise modern technology allows to establish $[11,12]$ some peculiarities in the width and position of the first peak of $S(q)$ and in $S(0)$ value, but it is just an indication of changes. So there is no clarity in the understanding of the microscopic processes which explain the whole spectrum of properties in these materials.

In this work we present results of structural analysis of 37 atomic models of different metals, restored from exper-

\footnotetext{
a e-mail: sas@physics.susu.ac.ru
}

imental data. Comparison of changes in atomic structure and features of different experimental data was done.

\section{Computational details}

We study the atomic structure of liquid metals numerically restored from scattering data by means of investigating local tetrahedral configurations of the atoms. Experimental data for $\mathrm{Cs}, \mathrm{Rb}, \mathrm{Hg}$ was taken along the liquidvapor coexistence curve and covers the whole region of liquid phase existence. Data for $\mathrm{Cu}, \mathrm{Fe}, \mathrm{Au}$ was obtained near the corresponding melting point. Atomic models construction was done by Reverse Monte-Carlo and Schommers methods [13] in our early works and works of other authors. Detailed description of experimental data and models is shown in table 1. Besides the metallic systems, several tens of LJ models with different temperatures and densities were constructed and investigated in the same way.

\begin{tabular}{|c|c|c|c|c|}
\hline & $\begin{array}{c}\mathrm{S}(\mathrm{k}) \\
\text { source }\end{array}$ & $\begin{array}{c}\text { Model } \\
\text { source }\end{array}$ & $\begin{array}{c}\text { Temp. } \\
\text { range. K }\end{array}$ & $\begin{array}{c}\text { Number } \\
\text { of models }\end{array}$ \\
\hline $\mathrm{Cs}$ & {$[1]$} & {$[14]$} & $323-1923$ & 9 \\
$\mathrm{Rb}$ & {$[11]$} & {$[15]$} & $373-1873$ & 9 \\
$\mathrm{Hg}$ & {$[16]$} & {$[17]$} & $293-1723$ & 5 \\
$\mathrm{Fe}$ & {$[18]$} & {$[19]$} & $1823-2033$ & 4 \\
$\mathrm{Ga}$ & {$[18]$} & {$[20]$} & $293-1273$ & 6 \\
$\mathrm{Au}$ & {$[21]$} & {$[21]$} & $1353-1853$ & 4 \\
\hline
\end{tabular}

Table 1. Data for analysis

We use Delaunay tessellation [22] for the analysis of the atomic structure of the models. A 2D illustration of tessellation and analysis is shown on fig. 1. The main idea of analysis is constructing simplicial voids (structural element of interatomic space) and looking after their fusion. The number of overlaps between simplicial voids $\mathbf{n}$ was used as a numeric criterion of looseness of the model [23]. Simplexes with large $\mathbf{n}(3,4$ in $3 \mathrm{D}$ case $)$ could be classified 
EPJ Web of Conferences

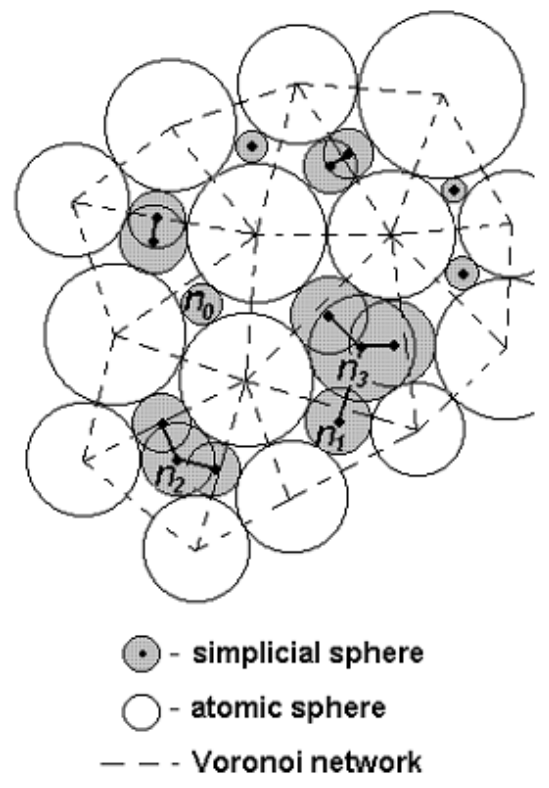

Fig. 1. A 2D representation of Delaunay tessellation and n parameter obtaininig.

as relatively loose aggregates of spheres atypical for crystals and dense liquids and simplexes with small $\mathbf{n}(0,1,2$ in $3 \mathrm{D}$ case) is a part of dense structure. According to the majority of simplexes we could relate the model to dense (I), porous (III) or intermediate (II) type.

\section{Results}

In all models we observed the same tendency: the number of loose tetrahedra is increasing with temperature rising and density falling. At some condition their number becomes dominant and the material becomes porous on atomic level. The interatomic space of loose structure contains large holes compatible with the atomic size [24].

Several slabs of Cs models, corresponding to different temperatures and densities are shown on fig. 2. Void arrangement is shown on the top line of slabs, and atomic arrangement is shown on bottom line. The right slabs represents heated (randomly distorted) BCC crystal.

It could be seen from fig. 2, that dense liquid is similar to heated crystal, but heated liquid has loose structure with large interatomic space. The qualitative explanation of that fact simply followed from the known idea about non-uniform expansion of simple liquids [1], but the numerical criterion was not proposed earlier. We could make a semiquantitative separation between dense and loose liquid, according to our method of Delaunay tessellation analysis. For Caesium the transition region (II) as well regions with dense (I) and loose (III) liquid are also shown on fig. 2.

As we said, all models (metals and LJ) had basically the same transformation of local atomic arrangement during expansion, but the positions of transition are different. Boundaries between regions with different structures are

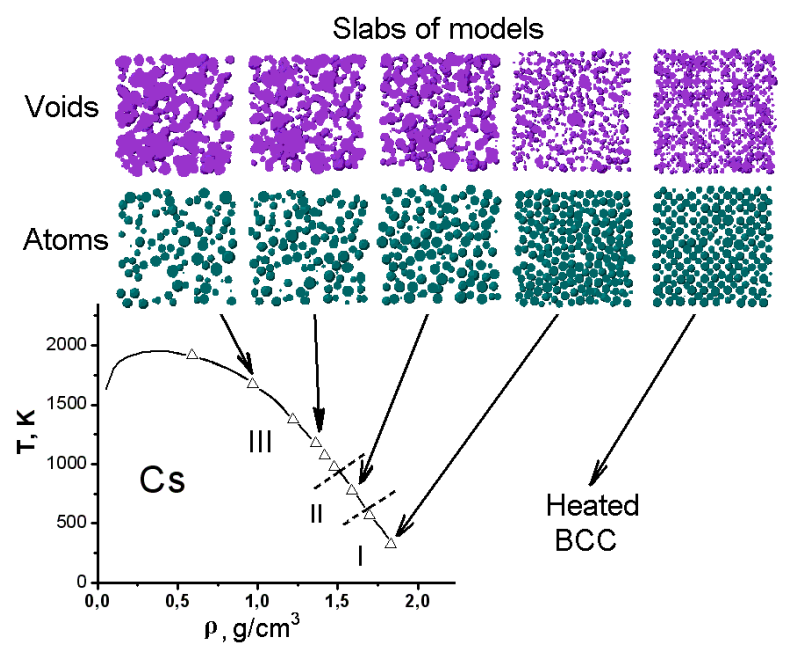

Fig. 2. Caesium models.

\begin{tabular}{|c|c|c|c|c|c|c|}
\hline & \multicolumn{2}{|c|}{$\begin{array}{c}\text { Critical } \\
\text { point }\end{array}$} & \multicolumn{2}{c|}{ I - II } & \multicolumn{2}{c|}{ II - III } \\
& $\mathrm{T}, \mathrm{K}$ & $\rho, \mathrm{gcm}^{-3}$ & $\mathrm{~T}, \mathrm{~K}$ & $\rho, \mathrm{gcm}^{-3}$ & $\mathrm{~T}, \mathrm{~K}$ & $\rho, \mathrm{gcm}^{-3}$ \\
\hline $\mathrm{Cs}$ & 1924 & 0.38 & 590 & 1.66 & 950 & 1.46 \\
$\mathrm{Rb}$ & 2017 & 0.29 & 820 & 1.32 & 1235 & 1.12 \\
$\mathrm{Hg}$ & 1751 & 5.8 & 1185 & 11.21 & 1680 & 9.17 \\
$\mathrm{Fe}$ & - & - & $2150^{*}$ & - & $2400^{*}$ & - \\
$\mathrm{Ga}$ & - & - & 1300 & - & $1700^{*}$ & - \\
$\mathrm{Au}$ & - & - & $1945^{*}$ & - & $2100^{*}$ & - \\
\hline $\mathrm{LJ}^{* *}$ & 1.08 & 0.32 & 0.95 & 0.63 & 1.01 & 0.56 \\
\hline
\end{tabular}

Table 2. Borders beetwing regions with different atomic structures along the liquil-vapour coexistence curve. ${ }^{*}$ - extrapolated data, ** - in LJ units)

given in table 3. Experimental data for $\mathrm{Fe}, \mathrm{Au}, \mathrm{Ge}$ cover only part of liquid phase region, so estimation was done. It could be seen from table 3 , that the structural transformation is closer to the critical point in $\mathrm{Hg}$ and farther in alkali metals. Apparently, it is a sequence of intrinsic chemical bonding of matter and it has the same nature as the different symmetry of the coexistence curves of the materials [25]. So the same character of atomic structure transformation does not exclude the uniqueness of every material.

\section{Discussion}

Regions with different type of intrinsic structure have shown different properties. A large number of peculiarities in properties was found in the region where the atomic structure is changed. Qualitative changes were observed in susceptibility of $\mathrm{Cs}, \mathrm{Rb}$ [5], Knight shift of $\mathrm{Hg}$, Cs [7,6], adiabatic thermal pressure coefficient of Cs [10] , adiabatic compressibility of $\mathrm{Hg}[8]$ and so on.

The Knight shift data of $\mathrm{Hg}$ [7] (fig. 3) shows the presence of three regions $\left(13.6 \mathrm{~g} / \mathrm{cm}^{3}<\rho<11.0 \mathrm{~g} / \mathrm{cm}^{3}, 11.0\right.$ $\left.\mathrm{g} / \mathrm{cm}^{3}<\rho<9.2 \mathrm{~g} / \mathrm{cm}^{3}, 9.2 \mathrm{~g} / \mathrm{cm}^{3}<\rho\right)$ with different slopes. On the base of the analysis of electrical conductivity, Hall coefficient and Knight shift data Cohen et. al. [26] made the conclusion that these three regions correspond to 


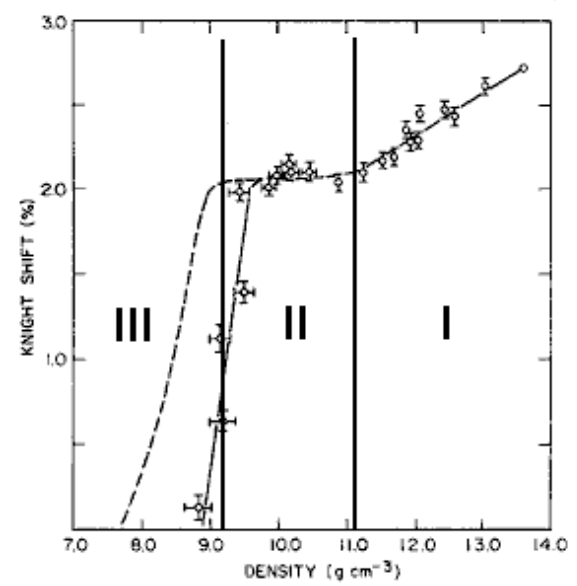

Fig. 3. Knight shift for Hg from [7]. Regions with different structure is shown.

different regimes of electrical conductivity. These regions coincide with regions of structure transformation (see tab. 3). Resently Tamura et. al. [27] have found that there exist medium-range fluctuations accompanying the metal-nonmetal transition in fluid $\mathrm{Hg}$ at $\rho \approx 9.2 \mathrm{~g} / \mathrm{cm}^{3}$, most likely it is a feature of loose liquid.

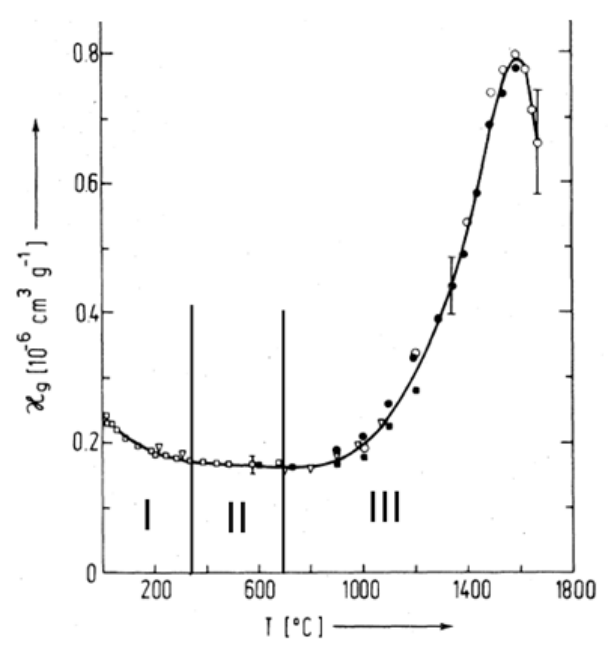

Fig. 4. Susceptibility of Cs from [5]. Regions with different structure are shown.

Some more interesting results were obtained for the magnetic suscebtibility of Cs [5] (fig. 4). There are four regions with different slopes. It is interesting, that three of them correspond to regions where the atomic structure changes. The Knight shift of Cs change the sign of the slope [6] at near $1.5 \mathrm{~g} / \mathrm{cm}^{3}$. Common observation of the electronic conductivity and the Hall coefficient leads to the conclusion [28], that the free electron approximation and Ziman formula for conductivity is broken at the densities lower than $1.3 \mathrm{~g} / \mathrm{cm}^{3}$. All these facts indicate, that loose liquid differ from porous one.

In molecular liquids it was found, that change in atomic structure correlates with disappearance of anomalous sound

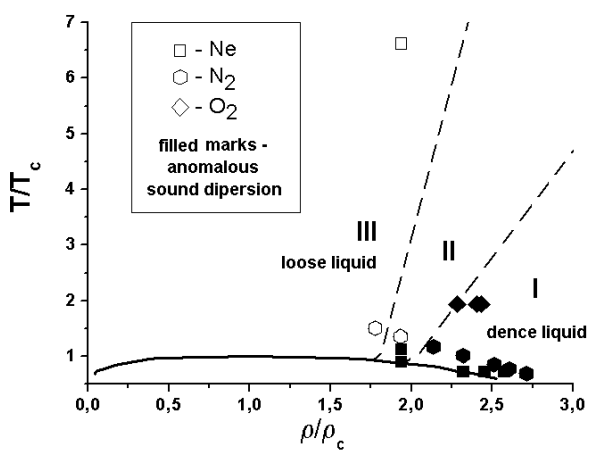

Fig. 5. Part of LJ phase diagram with regions of dense (I), porous (III) and intermediate (II) structure of liquid. Data of sound dispersion from [29].

dispersion [29]. On the figure 5 phase diagram of LJ liquid with the region of transition is shown. Filled marks correspond to the systems with anomalous sound dispersion, open marks are the systems without it. We can see from Fig. 5, that three regions of atomic strucrure exist in liquid phase as well as in supercritical fluid phase. Dense supercritical fluid like dense liquid has anomalous sound dispersion, but the loose one does not. So it could be an indicator of presence of difference between gas-like and liquid-like supercritical fluids.

\section{Conclusions}

Intrinsic structure of liquids topologically changes with heating and expansion.The comparison of the present results with experimental data obtained for several atomic and electronic properties of liquid metals allows us identify the region of transition as the relevant edge between dense liquid and loose liquid. Structure and properties of dense liquid are similar to disordered crystal, but expanded liquid has unique structure and properties qualitatively different from both solid phase and gas phase. Large and percolated interatomic space could be a criterion for the determination of loose liquid state.

\section{Acknowledgements}

The work as supported by RFBR. No. 09-03-00584-a,0903-00069.

\section{References}

1. R. Winter et al., Ber. Bunsenges. Phys. Chem. 91(12), 1327 (1987)

2. L. Landau, G. Zeldovich, Acta Phys. Chem. USSR 18, 194 (1943)

3. F. Hensel, E.U. Franck, Rev. Mod. Phys. 40(4), 697 (1968)

4. F. Hensel, E. Marceca, W.C. Pilgrim, J. Phys.: Condens. Matter 102, 11395 (1998) 
5. W. Freyland, Phys. Rev. B 20(12), 5104 (1979)

6. W.W. Warren-Jr, G. Brennert, U. El-Hanany, Phys. Rev. B 39, 4038 (1989)

7. W.W. Warren-Jr, F. Hensel, Phys. Rev. B 26(10), 5980 (1982)

8. K. Kobayashi, H. Kajikawa, Y. Hiejima, T. Hoshino, M. Yao, Journal of Non-Crystalline Solids 353, 3362 (2007)

9. D. Ishikawa, M. Inui, K. Matsuda, K. Tamura, S. Tsutsui, A.Q.R. Baron, Phys. Rev. Lett. 93, 097801 (2004)

10. L.A. Blagonravov, S.N. Skovorod'ko, A.S. Krylov, L.A. Orlov, V.A. Alekseev, E.E. Shpilrain, Journal of Non-Crystalline Solids 277, 182 (2000)

11. K. Matsuda, K. Tamura, M. Inui, Phys. Rev. Lett. 98, 096401 (2007)

12. K. Tamura, K. Matsuda, M. Inui, Jornal of physics: condensed matter 20(11), 114102 (2008)

13. W. Schommers, Phys. Rev. A 28(6), 3599 (1983)

14. D.K. Belashenko, A.S. Ginzburg, M. Mendelev, Rus.J.Phys.Chem. 74, 669 (2000)

15. G. Vorontsov, A., A. Mirzoev, A., D. Kuts, R. Gelchinski, B., A. Mirzoev Jr, A., Journal of Physics: Conference Series 98, 042023 (2008)

16. K. Tamura, S. Hosokawa, Phys. Rev. B 58(14), 9030 (1998)

17. D. Belashenko, Hight Temp. Phys. (Rus.) 20, 240 (2002)

18. Y. Waseda, The structure of non-crystalline materials: liquids and amorphous solids (McGraw-Hill, 1980)

19. A.G. Vorontsov, D.K. Belashenko, Bulletein of South Ural State University (Rus.) 2, 96 (2005)

20. D.K. Belashenco, A.C. Ginzburg, Rus.J.Phys.Chem. 75, 885 (2001)

21. S.A. Petrova, Poseedings of XI "Structure and properties of liquid metals and cinders" p. 223 (2004)

22. A.G. Vorontsov, D.A. Kuts, Journal of Physics: Conference Series 98, 012004 (2008)

23. A.G. Vorontsov, A.A. Mirzoev, G.P. Vyatkin, Journal of Non-Crystalline Solids 353, 35103514 (2007)

24. A.G. Vorontsov, A.A. Mirzoev, B.R. Gelchinski, Russian Journalof Physical Chemistry 77(11), 1800 (2003)

25. M. Ross, F. Hensel, J. Phys.: Condens. Matter 8, 1909 (1996)

26. M.H. Cohen, J. Jortner, Phys. Rev. A 10(3), 978 (1974)

27. K. Tamura, M. Inui, K. Matsuda, D. Ishikawa, Jornal of Non-Crystalline Solids 353, 33483357 (2007)

28. W.C. Pilgrim, R. Winter, F. Hensel, J.Phys.: Condens. Matter 9, B183 (1993)

29. F. Gorelli, M. Santoro, T. Scopigno, M. Krisch, G. Ruocco, Phys. Rev. Lett. 97, 245702 (2006) 